\title{
Le développement des turbomachines hydrauliques de grandes puissances
}

\author{
The development of high power hydraulic turbomachines
}

\author{
S. Casacci, G. Caillot \\ Société Neyrpic, Grenoble
}

Les groupes hydro-électriques se caractérisent naturellement par des avantages d'exploitation exceptionnels : - excellent facteur d'utilisation (grande robustesse, bonne fiabilité) ;

- performances élevées (les rendements sur modèle réduit dépassent $94 \%$ pour les turbines du type Francis, destinées à équiper des chutes moyennes);

- rapidité de mise en service et de prise de charge; - souplesse d'utilisation.

Lorsque les caractéristiques hydrologiques, combinées au relief, offrent une énergie disponible importante, le recours à des turbo-machines de grandes dimensions permet d'ajouter aux qualités précédentes, l'intérêt d'une puissance disponible élevée. Les impératifs économiques conduisent ainsi à des machines de plus en plus puissantes. Les puissances unitaires atteignent maintenant $800 \mathrm{MW}$ pour les turbines et dépassent $500 \mathrm{MW}$ pour les turbines-pompes.

Conserver aux turbomachines leurs qualités traditionnelles tout en élargissant leur domaine d'application (pour certaines machines, les vitesses tangentielles en marche normale excèdent $130 \mathrm{~m} / \mathrm{s}$ ), réduire leurs dimensions tout en élevant le niveau des performances hydrauliques afin d'améliorer l'économie globale des projets, tels sont les défis qui ont été relevés avec succès ces dernières années. En outre, les qualités demandées ne se limitent pas aux caractéristiques mais concernent aussi la réduction des nuisances. Les exploitants apprécient de plus en plus le silence et les techniciens ne peuvent éluder les difficultés que pose la diminution du niveau des bruits, même en invoquant l'indulgence que devrait leur valoir l'ensemble des problèmes ardus auxquels ils sont confrontés.

Satisfaire à toutes ces exigences et poursuivre les progrès demandent un approfondissent des connaissances concernant plus particulièrement les écoulements permanents ou instationnaires dans les turbomachines et le comportement des structures de formes complexes en présence de milieux fluides en mouvement. L'accroissement des puissances unitaires, donc des dimensions, entraîne un abaissement des fréquences propres et une augmentation des risques de résonance, d'où la nécessité d'une analyse approfondie du comportement dynamique des machines. Parallèlement à cela, cette progression n'a été rendue possible que grâce à l'évolution des procédés de fabrication tels que des techniques de soudage adaptées aux exigences nouvelles que pose la réalisation de grandes structures mécano-soudées.

Après avoir précisé, grâce à quelques chiffres, le domaine de quelques machines typiques, nous évoquerons les axes majeurs des travaux nécessaires pour aboutir à la réalisation de turbines hydrauliques de grandes puissances, performantes et sûres.

1. Groupes hydro-électriques de grande puissance - quelques chiffres

Quelques données caractéristiques permettent de préciser l'ampleur des problèmes à résoudre. Examinons donc rapidement les grandes familles de machines concernées.

\subsection{Groupes Francis pour chute moyenne}

L'exemple utilisé concernera la centrale d'ITAIPU (Brésil) qui est équipée de 18 groupes travaillant sous une chute de $126 \mathrm{~m}$ et ayant les caractéristiques suivantes:

Puissance nominale : $740 \mathrm{MW}$

Vitesse en marche normale: $91 \mathrm{tr} / \mathrm{min}$

Débit nominal : $625 \mathrm{~m}^{3} / \mathrm{s}$

Parmi les sous-ensembles du groupe, citons:

- l'amenée (bâche - avant-distributeur) :

diamètre d'entrée $\phi 9,64 \mathrm{~m}$, encombrement extérieur

$28 \times 25 \mathrm{~m}$, masse voisine de 1000 tonnes;

LA HOUILLE BLANCHE/N $\mathrm{N}^{\circ} 7 / 8-1983$ 
- la roue :

hauteur $=4,5 \mathrm{~m}$, diamètre extérieur $\phi 8,6 \mathrm{~m}$, masse voisine de 300 tonnes;

- l'arbre turbine :

longueur $=5,52 \mathrm{~m}$, diamètre au droit $\mathrm{du}$ palier ф3,2 $\mathrm{m}$;

- le rotor alternateur:

diamètre d'alésage $\phi 16 \mathrm{~m}$, masse du rotor environ 2500 tonnes.

\subsection{Groupes Francis et Francis réversible monoétage pour chute importante}

L'illustration concerne les groupes réversibles de REDENAT (France), étudiés pour EDF. Il s'agit de 4 pompes-turbines de $390 \mathrm{MW}$ sous $318 \mathrm{~m}$ de chute, à $300 \mathrm{tr} / \mathrm{min}$ :

- l'ensemble bâche - avant-distributeur a une masse de l'ordre de 260 tonnes, avec des soudures de $130 \mathrm{~mm}$ d'épaisseur à effectuer au chantier ;

- la roue, en acier inoxydable $17 / 4$ massif, a une masse de plus de 50 tonnes pour un diamètre extérieur de $5,4 \mathrm{~m}$;

- l'effort axial résultant, appliqué sur le flasque supérieur dans les conditions les plus sévères de service, dépasse $16.10^{6} \mathrm{daN}$;

- le rotor alternateur a une masse de l'ordre de 425 tonnes.

\subsection{Groupes réversibles multiétage pour très haute chute (au-delà de $1000 \mathrm{~m}$ )}

Citons quelques caractéristiques des machines du CLOU (France), étudiées pour EDF.

Il s'agit de groupes réversibles réglables (2 distributeurs synchronisés) de $300 \mathrm{MW}$ sous $1050 \mathrm{~m}$, à $600 \mathrm{tr} / \mathrm{min}$. Compte tenu des efforts considérables à reprendre, l'épaisseur des avant-directrices représente une obstruction de près de $40 \%$ du canal hydraulique. Par ailleurs, l'effort maximal sur les flasques atteint $20 \times 10^{6}$ daN. Quatre paliers sont nécessaires pour maintenir la ligne d'arbres, leur flexibilité ne doit pas excéder 5 à $6 \cdot 10^{-10} \mathrm{~m} / \mathrm{N}$ pour assurer au groupe un comportement dynamique satisfaisant.

\section{Conception hydraulique}

Les conceptions hydraulique et mécanique d'une machine sont indissociables car il convient de trouver des solutions permettant d'obtenir simultanément d'excellentes conditions de performance, de réalisation et d'exploitation. En outre, avec l'accroissement des dimensions, lié à celles des puissances unitaires, qui conduit à une réduction relative des rigidités des structures, des phénomènes hydro-élastiques peuvent apparaître et conduire à des fissurations de fatigue dans certains composants.

L'aspect hydraulique des développements s'appuie donc sur un approfondissement des connaissances sur les écoulements dans les turbomachines et sur les phénomènes de cavitation, ceci afin d'une part, de réduire les dimensions tout en améliorant les performances et d'autre part, de disposer de données sûres concernant les efforts hydrodynamiques permanents et pulsatoires, appliqués aux structures fixes et aux parties tournantes.

\section{Progrès effectués dans l'amélioration des tracés}

L'économie d'ensemble des installations de très grandes puissances, équipées de machines géantes, oblige les constructeurs à rechercher une réduction des dimensions.

Ces dimensionnements économiques s'obtiennent par réduction du cadre hydraulique (bâche, distributeur), augmentation de la puissance spécifique et adoption de vitesse de rotation élevée. Ces turbines géantes sont donc toujours des machines de hautes performances hydrauliques, tant du point de vue des rendements que de la cavitation.

Nous illustrons ce propos en comparant trois aménagements équipés de turbines Francis sous des chutes voisines de $60 \mathrm{~m}$ et donc les caractéristiques sont:

\begin{tabular}{|l|ccc|}
\cline { 2 - 4 } & \multicolumn{4}{c|}{ Centrale de } \\
\cline { 2 - 4 } & $\begin{array}{c}\text { Mequinenza } \\
\text { (Espagne) }\end{array}$ & $\begin{array}{c}\text { Inga 2 bis } \\
\text { (Zaïre) }\end{array}$ & $\begin{array}{c}\text { Tucurui } \\
\text { (Brésil) }\end{array}$ \\
\hline $\begin{array}{c}\text { Date d'élaboration } \\
\text { du tracé hydraulique }\end{array}$ & 1958 & 1974 & 1978 \\
\hline$\emptyset$ roue & 4300 & 6050 & 8150 \\
\hline Puissance maxi $(\mathrm{MW})$ & 80 & 175 & 360 \\
\hline Chute & 62 & 60 & 60 \\
\hline Vitesse & 150 & 107 & 81,8 \\
\hline$n_{s}$ approximatif & 300 & 320 & 330 \\
\hline
\end{tabular}

\subsection{Augmentation de la vitesse spécifique}

Dans le cas présent, les puissances spécifiques ont peu varié mais par contre, les vitesses spécifiques se sont notablement élevées: $n_{s} 300$ est devenu $n_{s} 330$ utilisé sous une chute de fonctionnement légèrement plus forte.

\subsection{Réduction des dimensions du cadre hydraulique}

Cet effort se traduit par des gains économiques concernant :

- l'encombrement extérieur de la bâche et par suite une diminution de longueur de la centrale, avec les gains de génie civil qui en découlent;

- certaines structures propres de la turbine: bâchespirale, avant-distributeur, flasques, avec une répercussion directe sur leur masse.

Les progrès accomplis s'illustrent parfaitement à l'aide du dessin comparatif des tracés respectifs des trois machines étudiées (Fig. 1 et 2 ). On remarque en outre que cet effort s'est accompagné :

= d'une réduction de la longueur relative du guidage de la grille avant-distributrice (rapport de la longueur des avant-directrices à leur espacement);

- d'angles d'attaque importants ; 


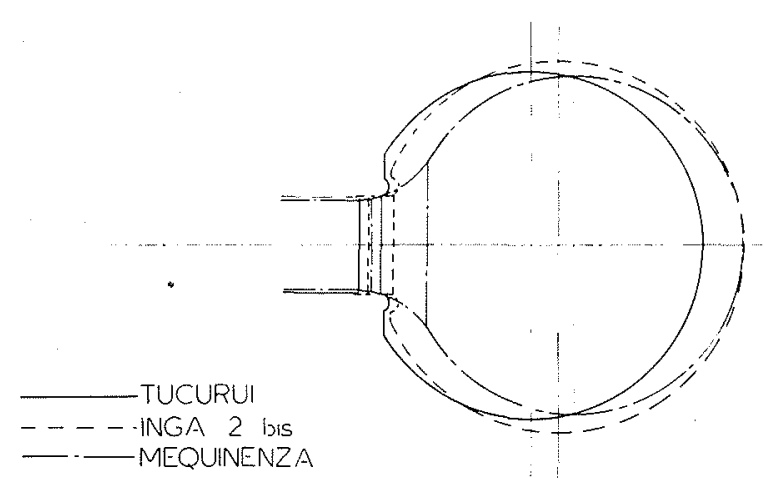

Figure 1. - Tracé hydraulique comparatif des bâches (section mériđienne)

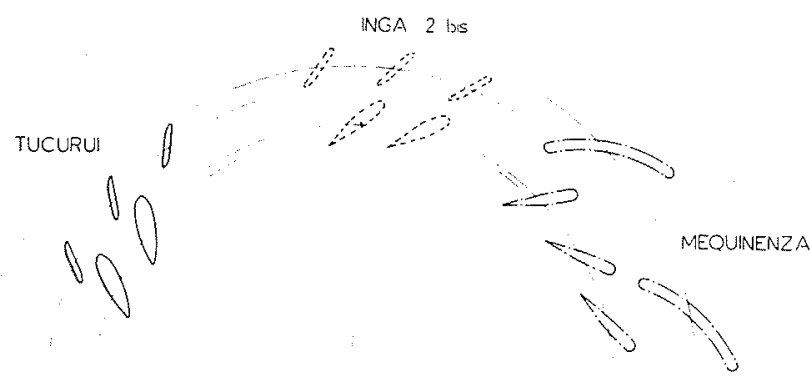

Figure 2. - Tracé hydraulique comparatif de la double grille avant-distributeur - distributeur

- d'une imbrication très nette de la double grille constituée par l'avant-distributeur et le distributeur, avec des sections de directrices très supérieures à celles des avantdirectrices.

soit un ensemble de problèmes qu'il a fallu résoudre pour pouvoir retenir en toute sécurité ce faible niveau d'encombrement.

\subsection{Rendements}

On pourrait croire que le rendement des turbomachines de grande puissance a été légèrement sacrifié aux dépens des efforts de rentabilité précédents. Cela n'est pas le cas, bien au contraire, puisque grâce aux recherches effectuées, la réduction du cadre hydraulique des machines de Tucurui s'est accompagnée d'une amélioration de $1 \%$ du rendement modèle par rapport à Inga 2 bis.

\subsection{Sécurité de fonctionnement, cavitation et connais- sance des sollicitations hydrodynamiques}

$\mathrm{Si}$ la conception mécanique des machines doit prendre en compte les amplitudes des efforts hydrauliques pulsatoires, la conception hydraulique doit les réduire au minimum. La diminution de l'amplitude des perturbations hydrodynamiques oriente vers des profils nombreux et effilés, tandis que le souci de dimensionner les profils pour que leurs fréquences propres se situent au-dessus du domaine des amplitudes maximales des perturbations conduit à des profils épais. D'autres contraintes mécaniques, liées à l'aspect fabrication, sont également à considérer. Les tracés de turbo- machines sont donc le fruit d'incessants compromis entre l'hydraulique et la mécanique. Citons quelques exemples de compromis dans la conception des turbines de grandes dimensions:

- l'épaississement des profils de directrices pour permettre le tourillonnement seulement sur deux paliers; - la réduction du nombre des aubes des roues (15 à 17 aubes autrefois, pour 11 à 13 maintenant) pour permettre d'utiliser des profils présentant des dimensions plus importantes et offrant une plus grande sécurité visà-vis des vibrations;

- le choix des épaisseurs de bord de sortie des aubes qui doivent rester suffisamment minces pour conserver de très bonnes performances mais en même temps résister aux sollicitations dont ils sont l'objet;

- une variation d'épaisseur des aubes de la roue suivant des lois d'évolution à simple courbure pour permettre, si ce mode de fabrication est retenu, un matriçage à partir d'une tôle mère fraisée sur une seule face, etc.

La sécurité de fonctionnement des turbines de grandes dimensions suppose une connaissance approfondie des sollicitations hydrodynamiques. Il s'agit en particulier :

- de l'évaluation du comportement dynamique en service des structures fixes et mobiles, afin de prévenir les risques de vibrations dangereuses et de procéder à un dimensionnement correct des structures concernées; - de la connaissance des instabilités d'écoulement et de leurs manifestations, non seulement dans la turbomachine et à l'aval immédiat de la roue mais également dans l'ensemble de l'aménagement hydro-électrique, incluant conduites forcées et rameaux d'évacuation, ce dernier point restant généralement à la charge du Maître d'Ceuvre ;

- des prévisions des phénomènes de cavitation, en tenant compte des variations de chute consécutives au marnage des retenues. En outre, signalons l'acuité toute particulière des phénomènes d'usure dans le cas des très hautes chutes. Les grandes vitesses d'écoulement accentuent les éventuelles pointes de dépression génératrices de cavitation. De plus, la vitesse d'érosion est pratiquement proportionnelle à la puissance cinq de la vitesse relative de l'écoulement par rapport à la paroi. Aussi peut-on se rendre compte de la difficulté que pose la cavitation pour les très hautes chutes et de la nécessité d'une étude théorique poussée des écoulements, des vérifications sur modèle réduit à effectuer, et de la très grande conformité par rapport à celui-ci que doit présenter la machine industrielle. En outre, comme les contraintes dans le métal dépendent directement de la hauteur de chute, les épaisseurs des avant-directrices, directrices et aubes de la roue vont en s'accroissant, entraînant ainsi une altération plus ou moins grande des caractéristiques et des performances. Les effets pulsatoires croissent comme la hauteur de chute sur la roue et prennent des valeurs très élevées pour les très hautes chutes.

La connaissance précise de la répartition des pressions le long des aubages constitue donc un facteur important de progrès dans la conception des turbomachines.

Les recherches théoriques doivent nécessairement aboutir à des outils mathématiques exigeant des volumes 
de calculs acceptables, ce que permettent par exemple les approches quasi tridimensionnelles. Ces programmes consistent à modéliser, en première approximation, les conduits hydrauliques par un ensemble de filets fluides en équilibre radial. Avec ces données, on calcule ensuite l'écoulement dans la machine élémentaire constituée par la grille d'aubes tracée sur les surfaces de révolution caractérisant le filet fluide correspondant. Ces résultats servent ensuite à définir une nouvelle approximation du réseau d'écoulement méridien jusqu'à convergence de la solution. Par itération, on obtient une représentation de l'écoulement dans les organes d'alimentation, le rotor et l'évacuation de la turbomachine.

Ces méthodes font appel à deux programmes couplés (cf. [1]):

- calcul de l'écoulement, en général rotationnel, dans les conduits axisymétriques, l'effet de la grille d'aubes est représenté par un champ de forces judicieusement choisi;

- calcul de l'écoulement dans une grille d'aubes tracée sur une surface de révolution de méridienne quelconque.

Le problème est résolu pour un fluide parfait et incompressible dont l'écoulement, considéré stationnaire par rapport aux aubages, est partagé entre un écoulement principal sans frottement et un écoulement pariétal avec frottement du type couche limite. Ces pertes sont simulées par introduction d'un champ de force approprié dans les équations de la mécanique.

Parallèlement à ces programmes déjà opérationnels, d'autres équipes de chercheurs s'attaquent à l'étude des écoulements tridimensionnels de fluides réels dans les turbomachines. Les méthodes en cours de développement nécessiteront des temps de calculs importants qui feront probablement que leur emploi restera limité à la qualification de programmes plus simples ou à des recherches spécifiques.

Tous ces progrès diminueront le recours à l'expérience mais ne le supprimeront pas, ils permettront de construire des essais en vue d'un contrôle des hypothèses. La philosophie des aller et retour de la théorie à l'expérience restera toujours une nécessité.

L'approche des fluctuations de pression relève encore du domaine expérimental car seule l'expérimentation sur turbine industrielle et sur modèle réduit permet actuellement de connaître les amplitudes et fréquences des sollicitations ainsi créées. Les modèles réduits peuvent alors comporter la mesure des contraintes sur les parties fixes et mobiles et une étude assez précise de ces phénomènes fluctuants se fait à l'aide d'enregistrements magnétiques permettant une analyse poussée des signaux enregistrés.

Ces études dynamiques sont très importantes afin d'éviter que les sollicitations hydrodynamiques pulsatoires soumettent les organes de la turbine à des cycles de contraintes alternées de fréquence voisine de celle des fréquences propres des structures composantes.

\section{Conception mécanique}

\subsection{Structures du groupe}

Les problèmes de conception diffèrent selon les com- posants. En effet, outre l'aspect du dimensionnement, rendu délicat par la complexité des formes et du chargement hydrodynamique, il faut répondre aux impératifs de réalisation, en partie imposée sur le site pour certains éléments par suite des limitations de gabarit de transport, et, adopter des conceptions permettant un entretien aisé, mais aussi rare que possible.

\subsubsection{Dimensionnement des structures mécaniques}

Compte tenu des enjeux liés à un dimensionnement satisfaisant des structures complexes des turbines, NEYRPIC a toujours suivi de très près les progrès effectués en la matière. La conception et la construction des turbomachines de grandes dimensions ont donc bénéficié des progrès notables dans le domaine du calcul statique et dynamique des structures, notamment par le développement de la méthode des éléments finis. Afin de répondre à ses besoins propres, NEYRPIC a déve. loppé dans les années 70 un certain nombre de logiciels de calculs parmi lesquels nous citerons:

- pour les programmes généraux (élasticité linéaire)

- dans le domaine statique:

NEYPLAX (1970): élasticité bidimensionnelle (plan, révolution)

NEYCOQUE (1972): élasticité coque de révolution

NEYSTRUC (1972): structures 3 dimensions (poutres et coques)

NEYTRIC (1974): élasticité 3 dimensions (élément massif)

- dans le domaine dynamique :

NEYOMEGA (1978): structures 3 dimensions (poutres et coques), calcul des fréquences propres, réponses sismiques et réponses à des excitations aléatoires

- pour les roues de turbines hydrauliques:

- étude statique par NEYROUEF (1974) et dynamique par NEYROUDY (1979).

Les besoins très spécifiques, non couverts par les logiciels précédents, peuvent être satisfaits en utilisant les grands codes de calcul disponibles (NASTRAN, CASTEM (Aquamode), BOSOR, INCA, TRICO, etc.) à l'exploitation desquels, certains de nos ingénieurs sont formés.

Ceci étant, nous allons examiner certains problèmes de dimensionnement typiques des quelques grands composants de turbine de forte puissance.

\subsubsection{Avant-distributeur et bầche}

Cet ensemble constitue l'un des composants fondamentaux d'une turbomachine hydraulique. Outre ses nécessités hydrauliques d'alimentation ou de diffuseur, il se caractérise par les traits essentiels suivants:

- structure de liaison et d'ancrage de la turbomachine dans le génie civil. C'est l'organe dans lequel vont transiter les efforts provenant des autres parties de la turbine. De sa tenue dépend le comportement des pièces fixes et tournantes de la machine,

- élément de plus grande dimension de la turbine, conditionnant l'encombrement de la machine à ce niveau, avec les répercussions économiques que cela implique. 
Ces fonctions doivent s'accompagner de performances hydrauliques élevées, nécessitant un profilage adéquat des avant-directrices. Ces dernières, placées au sein de l'écoulement, sont soumises à des sollicitations complexes liées aux fluctuations de portance qu'elles peuvent subir. Parallèlement, elles assurent une fonction d'entretoise entre les deux côtés du canal hydraulique. S'agissant d'une structure devant reprendre des efforts importants, elle présente une grande rigidité naturelle qui la rend en général peu sensible aux perturbations de faible amplitude au niveau de l'écoulement. Toutefois, pour les machines de grandes dimensions, travaillant sous chute peu élevée, ensemble de facteurs conduisant à un abaissement des valeurs des fréquences propres, la réponse aux sollicitations dynamiques doit être étudiée avec soin afin d'éviter tout phénomène d'instabilité hydro-élastique (cf. [2]).

L'étude statique du comportement de l'avant-distributeur sous l'action des flasques et de la bâche est conduite, lorsqu'il s'agit de structures mécano-soudées construites à partir de tôles, à l'aide du programme NEYSTRUC. Ce programme traite du comportement tridimensionnel d'éléments de plaques triangulaire et rectangulaire.

Le chargement pris en compte est également tridimensionnel. L'avant-distributeur étant assimilé à une structure cyclée, seul un pas de structure comprenant une avant-directrice et les morceaux d'anneaux correspondants est à considérer. La condensation de la structure étudiée n'introduit aucune approximation car le programme prend automatiquement en compte les conditions aux limites permettant de relier les efforts et les déformations des deux sections latérales limitant le pas.

Cette étude permet d'analyser le champ des contraintes statiques en charge (voir Fig. 3).

Aux sollicitations quasi statiques exercées par la volute et les flasques, peuvent se superposer des sollicitations hydrodynamiques pulsatoires au niveau des profils.

$\mathrm{Si}$ on analyse les évolutions dimensionnelles des avant-distributeurs en fonction de la chute, on constate qu'à diamètre d'avant-directrice identique, lorsque la chute diminue, la hauteur du distributeur augmente et l'épaisseur des avant-directrices diminue. Ces dernières évolutions vont toutes les deux dans le sens d'un abaissement des fréquences propres. On conçoit dans ces conditions que les avant-directrices soient de plus en plus exposées à des contraintes vibratoires sous l'action de forces excitatrices pulsatoires liées soit à des décollements sur l'arête d'entrée, soit à des lâchers de tourbillons de Karman au niveau de l'arête de sortie.

Ces sollicitations pulsatoires ont pour origine les fluctuations de portance et de couple dues à l'écoulement le long de l'avant-directrice. Ces fluctuations, par suite d'un phénomène d'auto-excitation, s'attachent à une fréquence propre de l'avant-directrice (dans la pratique, le premier mode de flexion). Elles peuvent atteindre une amplitude suffisante pour provoquer des contraintes dynamiques importantes.

Rappelons que pour des cas simples tels qu'un cylindre placé dans un écoulement perpendiculairement à ses génératrices, il se forme dans le sillage du profil des
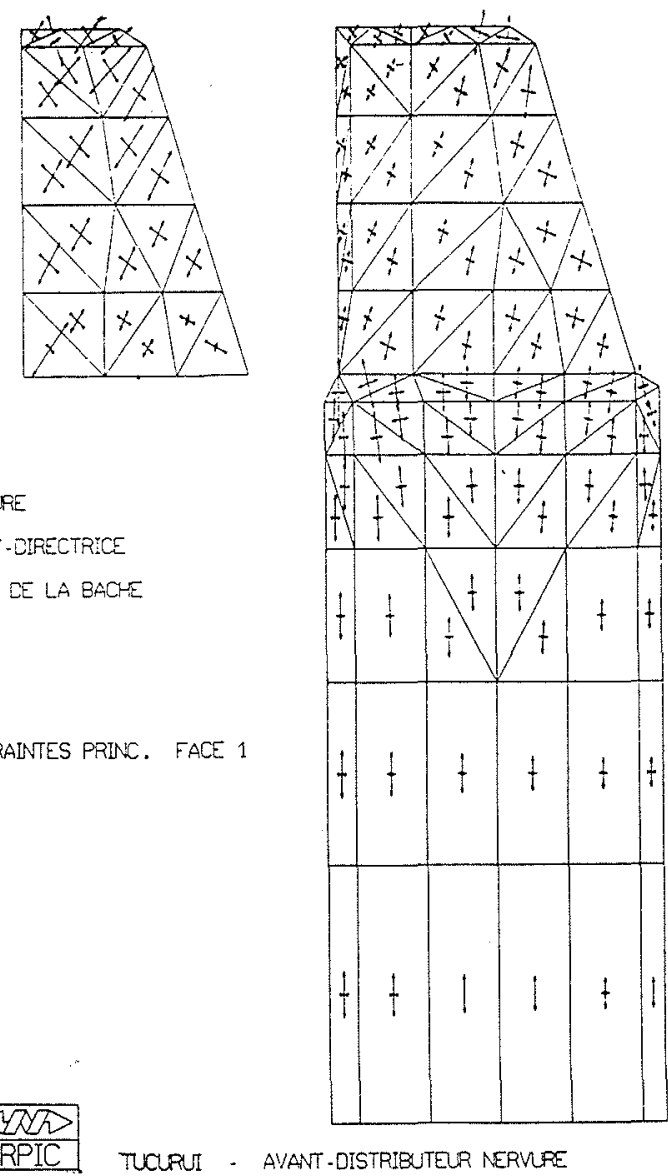

Figure 3. - Contraintes principales calculées par NEYSTRUC dans un avant-distributeur de turbine Francis de grandes dimensions

tourbillons réguliers et un spectre d'excitation se résu. mant à une fréquence unique. On peut alors relier la fréquence de lâcher des tourbillons $\left(f_{k}\right)$ à la vitesse d'écoulement $(V)$ et à la dimension transversale du profil $(e)$ par une relation du type :

$$
f_{k}=S V / e
$$

où $S$ représente le paramètre adimensionnel de Strouhal, constant pour des profils d'un même type en similitude géométrique.

Lorsqu'on passe à des formes de profil complexes telles que celles des avant-directrices, on relève plusieurs difficultés :

- la fréquence des lâchers de tourbillons ne dépend plus simplement de la dimension transversale du profil mais aussi de la forme générale du profil et du bord de fuite en particulier ;

- le spectre des excitations est généralement plus étalé et c'est ce que nous avons enregistré, lors d'essais récents, sur un modèle d'avant-directrice pour lequel nous avons analysé expérimentalement les fluctuations de vitesse dans le sillage immédiat du bord de fuite. Nous avons observé que tous les spectres présentaient globalement un palier jusqu'à un nombre Strouhal de 0,3 à 0,35 , puis une décroissance relativement linéaire en échelle logarithmique. Dans le cas d'un spectre étalé, on peut ainsi définir un nombre de Strouhal de réfé- 
rence en caractérisant la fréquence la plus élevée des sollicitations d'amplitude notable.

L'ampleur de la gravité du phénomène d'autoexcitation dépend:

- de la forme du profil de sortie des avant-directrices qui conditionne en partie la forme du spectre des excitations et leurs amplitudes;

- de la position de la fréquence propre de flexion par rapport au spectre des excitations.

Le dimensionnement des avant-distributeurs doit donc prendre en compte :

- les efforts statiques en service;

- la réponse dynamique des avant-directrices aux excitations engendrées par l'écoulement;

- les facteurs de concentration de contrainte liés aux formes de raccordement au niveau de l'attache de l'avant-directrice sur les anneaux ;

- la limite de fatigue du matériau dans l'eau.

En outre, les procédés de fabrication en atelier et sur le site doivent être adaptés pour réduire au minimum les contraintes internes et écarter les risques de microfissuration.

\subsubsection{Distributeur}

Le distributeur est constitué d'une part des directrices mobiles permettant de régler l'alimentation de la turbomachine et par suite de moduler sa puissance, et, d'autre part, des flasques formant canal hydraulique. Il est situé entre l'avant-distributeur et la roue.

Le dimensionnement de cet organe est particulièrement délicat pour les machines réversibles. En effet, en dehors des zones normales de fonctionnement, l'aubage de la roue des turbines-pompes n'étant pas adapté à l'écoulement provenant du distributeur (sens turbine), d'importantes fluctuations de pression se produisent entre la roue et les directrices (entrefer). Il en est de même dans le sens pompe, lorsque les directrices sont attaquées avec un grand écart d'incidence par l'écoulement provenant de la roue. Il en résulte, lors des fonctionnements transitoires, des fluctuations de pression internes qui engendrent:

- d'importantes fluctuations de couple sur les directrices mobiles;

- des oscillations de couple sur l'arbre moteur ;

- des fluctuations de poussées radiales qui s'exercent sur la roue.

En ce qui concerne les couples sur les directrices, les fluctuations sont maximales à l'emballement et lors des déclenchements en turbine et sont aggravées par le fait que pour ces fonctionnements, le couple moyen et la poussée résultante restent faibles, ce qui réduit d'autant l'amortissement naturel dû au frottement dans les paliers. Le spectre d'excitation contient des fréquences élevées qui correspondent en particulier au passage des aubes de la roue devant la directrice et à ses premiers harmoniques. Ces valeurs élevées de charges fluctuantes ont plusieurs conséquences. D'une part, elles nécessitent un surdimensionnement des organes de sécurité insérés dans la commande du vannage, ce qui majore d'autant l'effort maximal de manœuvre lors de la fermeture de la directrice sur un corps étranger. D'autre part, ces sollicitations exigent une étude approfondie des comportements statique et dynamique de la directrice.

Les études statiques sont conduites par éléments finis soit à partir de NEYSTRUC ou de NEYTRIC selon les types de structures. En ce qui concerne l'étude dynamique, elle consiste essentiellement à positionner les fréquences propres de la directrice entre les fréquences excitatrices afin d'éviter tout phénomène de résonance. L'immersion du profil dans le fluide est prise en compte par l'effet d'inertie ajoutée qui en résulte [3].

En outre, les flasques sont soumis à des efforts considérables pouvant atteindre $2010^{6} \mathrm{daN}$. Leur dimensionnement repose alors sur des études par éléments finis permettant d'analyser les sollicitations dans l'ensemble de la pièce, y compris les soudures d'assemblage et les nervures de raidissement.

\subsubsection{Roue}

La conception et la fabrication des roues sont incontestablement un des éléments fondamentaux de la réussite des turbines géantes. Ceci suppose la maîtrise des problèmes de dimensionnement de cet organe, c'est-à-dire, la connaissance des charges permanentes ou fluctuantes d'origine hydrodynamique d'une part, et, d'autre part, la possession de procédés de calculs statiques et dynamiques précis pour ce type de structure avec bien sûr en parallèle une évaluation correcte des limites de fatigue des matériaux utilisés.

Le calcul statique, effectué à l'aide de NEYROUEF, permet de déterminer le champ des contraintes dans l'aube, le plafond et la ceinture, à partir des efforts

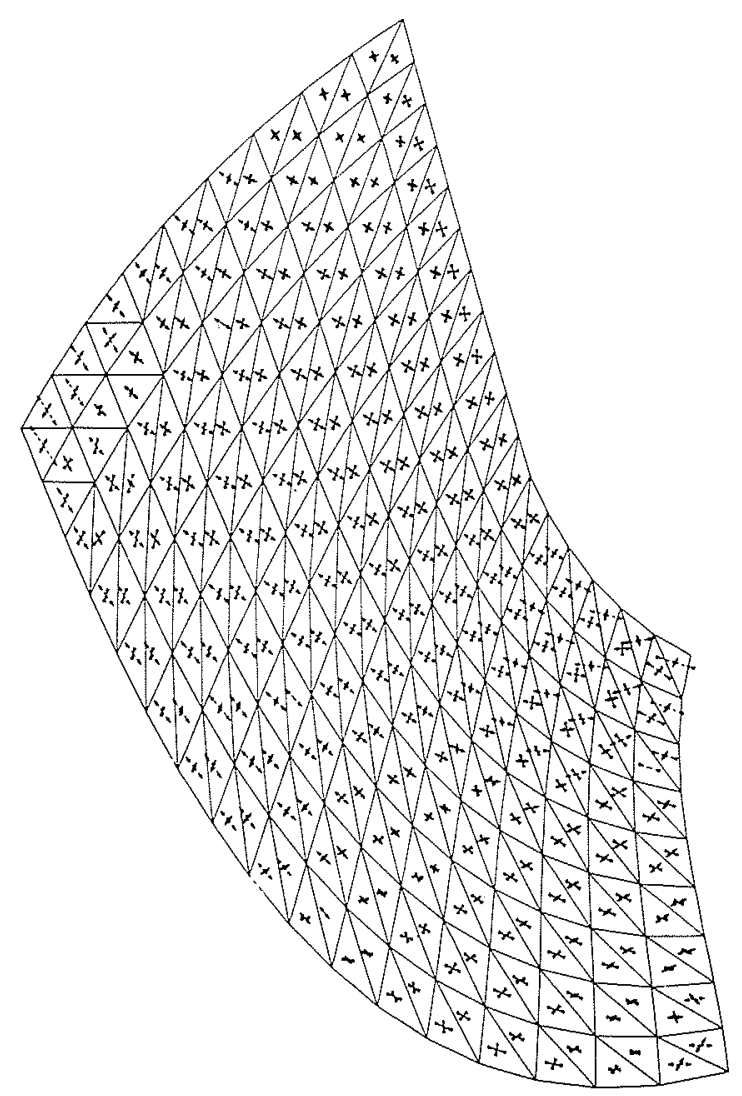

Figure 4. - Contraintes principales sur la face intrados d'une aube de roue Francis pour un fonctionnement à pleine charge, au synchronisme 
hydrodynamiques évalués à l'aide d'un calcul en grille d'aubes. Traditionnellement, ces calculs mettent en évidence deux zones de concentration des contraintes, l'une à l'attache de l'arête de sortie de l'aube sur le plafond, l'autre à la liaison de l'arête d'entrée de l'aube avec la ceinture.

Les calculs par éléments finis permettent alors de rechercher les modifications locales de structure (épaississement local, adaptation des formes de raccordement) ou globales (notamment formes du plafond) permettant de ramener ces concentrations à des valeurs compatibles avec la limite de fatigue des matériaux. Les figures 4 et 5 illustrent au niveau de l'aubage les contraintes et déformations calculées respectivement pour des fonctionnements au synchronisme et à l'emballement.

Le calcul dynamique, mis au point par Neyrpic (programme NEYROUDY), permet de déterminer, à l'aide d'un maillage moins serré que pour le calcul statique, les fréquences propres de la structure et ses modes de vibration. Rappelons que les fréquences propres de la roue doivent rester assez éloignées des fréquences perturbatrices, une résonance franche pouvant entraîner des amplitudes de vibrations sensibles, étant donné le faible coefficient d'amortissement réduit des matériaux utilisés $\left(10^{-3}, 10^{-4}\right)$. On constate toutefois que les fréquences propres les plus basses correspondent à des déplacements préférentiels de la ceinture, les modes faisant intervenir essentiellement les aubes apparaissant pour des valeurs de fréquences nettement plus élevées.

Signalons que le programme NEYROUDY a été validé en particulier à l'aide d'une étude comparative entre le

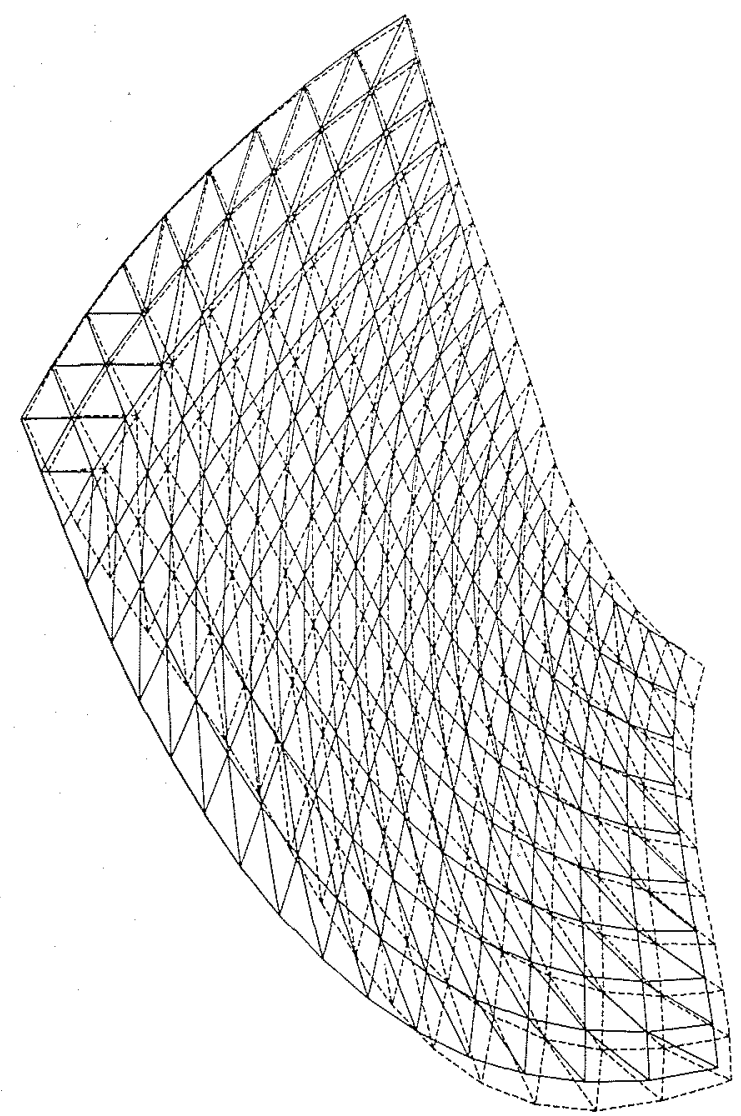

Figure 5. - Déformations à l'emballement d'une aube de roue Francis calcul et la mesure en atelier des fréquences propres d'une roue industrielle Francis de diamètre $\emptyset 4650 \mathrm{~mm}$ :

\begin{tabular}{|c|c|c|c|}
\hline \multirow{2}{*}{$\begin{array}{l}\text { Ordre } \\
\text { de la } \\
\text { fréquence } \\
\text { propre }\end{array}$} & \multirow{2}{*}{$\begin{array}{l}\text { Mode de } \\
\text { vibration }\end{array}$} & \multicolumn{2}{|c|}{ Valeur de la fréquence propre } \\
\hline & & $\begin{array}{l}\text { calculée par } \\
\text { NEYROUDY }\end{array}$ & mesurée \\
\hline 1 & $\begin{array}{l}4 \text { nouds sur } \\
\text { la ceinture }\end{array}$ & $48,08 \mathrm{~Hz}$ & $46 \mathrm{~Hz}$ \\
\hline 2 & $\begin{array}{l}6 \text { nouds sur } \\
\text { la ceinture }\end{array}$ & $83,87 \mathrm{~Hz}$ & $80,2 \mathrm{~Hz}$ \\
\hline 3 & $\begin{array}{l}8 \text { noeuds sur } \\
\text { la ceinture }\end{array}$ & $101,6 \mathrm{~Hz}$ & $101.7 \mathrm{~Hz}$ \\
\hline
\end{tabular}

\subsubsection{Réalisation}

\subsubsection{Avant-distributeur et bâche}

Par suite de ses dimensions et de sa masse, cet ensemble n'est jamais réalisable en atelier pour les turbines de fortes puissances. Il doit être construit en partie sur le site.

Pour ce type de machine, les efforts sont tels que les solutions par assemblage mécanique ne sont plus envisageables et qu'une construction mécano-soudée doit être exécutée au chantier. S'agissant de souder des tôles de forte épaisseur ou des pièces de très grandes dimensions, on conçoit que la réalisation de la structure pose de délicats problèmes de conception et de fabrication.

En règle générale, les avant-distributeurs sont réalisés en atelier en autant de parties que le permettent les conditions de transport. Les sous-ensembles ainsi préparés sont détensionnés avant expédition au chantier afin de réduire à un minimum les contraintes résiduelles au niveau des soudures particulièrement sollicitées dynamiquement. Les avant-distributeurs sont munis de bavettes, la soudure des volutes s'effectue en centrale. Les épaisseurs à souder peuvent atteindre $130 \mathrm{~mm}$, aussi, la réalisation de la bâche sur le chantier s'accompagne-t-elle de déformations en cours de soudage, puis de l'enrobage dans le béton. Dans ces conditions, il est nécessaire de reprendre sur le site, à l'aide d'un outillage d'usinage spécifiquement prévu, l'usinage des surfaces devant assurer l'appui des flasques et des étanchéités.

Un manque de rigueur dans les opérations de fabrication et de montage peut conduire à des fissurations en service. Outre la parfaite exécution des contrôles habituellement prévus (ressuage, magnétoscopie, ultrasons, radiographie locale si nécessaire, etc:), il est nécessaire de limiter au minimum les contraintes résiduelles car elles se superposent aux contraintes statiques sous charge et aux contraintes dynamiques, ainsi que les concentrations de contraintes. Dans cet esprit, des dispositions appropriées sont prises au niveau des gammes de fabrication pour limiter les bridages, des traitements thermiques pour contrôler les paramètres dans les parties les plus minces et les plus épaisses, de la finition des soudures avec par exemple le meulage partiel dans les zones sensibles des congés de raccordement des avant-directrices avec les anneaux de l'avant-distributeur. 


\subsubsection{Roue}

La réalisation des roues délivrant de fortes puissances pose toujours de délicats problèmes.

Pour les machines de haute chute, surtout lorsqu'il s'agit de turbines-pompes, les roues prennent des proportions de disque plat. Les canaux sont de forme peu accessibles. Le meulage des aubes, pour obtenir les profils correspondant à ceux du modèle, devient difficile hors des extrémités. Ces inconvénients, particulièrement marqués dans les turbines-pompes, vont jusqu'à conduire à une limite technique pour la valeur de la vitesse spécifique au-dessous de laquelle il n'est pratiquement pas possible de descendre. Cette valeur est actuellement de 26 environ, ce qui conduit au développement des turbines-pompes multicellulaires (dont les machines biétage réglables) pour des chutes supérieures à $1000 \mathrm{~m}$.

Dans le cas des turbines Francis de grandes dimensions, les problèmes sont différents. Il s'agit de pièces pouvant atteindre 300 tonnes, or, au-delà d'une masse que l'on peut fixer aux environs de 100 à 120 tonnes, il devient nécessaire d'abandonner la solution moulée en une partie.

En effet, même si la construction monobloc restait possible, les risques présentés par la coulée de telles pièces ne sont plus admissibles. Sur ces pièces de grandes dimensions, on a constaté ces dernières années des incidents graves tels que :

- rupture du plafond y compris le plateau d'accouplement;

- séparation quasi totale de la roue selon un plan horizontal par suite d'une interruption accidentelle de l'opération de coulée ;

- déformations ou déplacements des noyaux.

Par ailleurs, à l'accroissement des dimensions, correspond une diminution du nombre de fonderies capables de couler de telles pièces, d'où incompatibilité avec la recherche de solutions économiques et également avec la cadence de livraison requise (en général 3 à 4 mois entre groupes).

De ce fait, pour nos roues de grandes dimensions, nous adoptons une solution consistant à assembler par soudure des éléments séparés :

- plafond moulé soit lisse, soit avec attente d'aubes pour raccordement dans un plan horizontal;

- aubes moulées ou matricées ;

- ceinture lisse moulée.

La construction en éléments séparés présente de nombreux avantages:

- qualité de la construction: respect des épaisseurs et des dimensions, chacun des éléments peut en effet être usiné séparément ;

- qualité du métal qu'il est plus facile d'assurer sur des composants de masse inférieure;

- qualité de la similitude hydraulique: lors du montage des aubes, il est relativement facile d'assurer l'homologie avec le modèle: angles et ouvertures;

- simplification de l'équilibrage de la roue qui est construite à partir d'un plafond et d'une ceinture préalablement usinés et d'aubes que l'on dispose après pesée de manière à réduire le balourd au minimum ;

- possibilité d'emploi d'aciers de nuances différentes pour les diverses parties de la roue ;
- possibilité de construire la roue sur place si les conditions de transport interdisent la réalisation d'une roue monobloc dans les ateliers du constructeur.

La solution des aubes matricées, qui présente de nombreux avantages du point de vue de la qualité de réalisation, a été mise au point en collaboration avec une firme française; elle est couverte par des brevets.

Les aubes sont obtenues par formage à partir d'ébauche plane.

Il convient d'abord de déterminer les dimensions de la tôle capable de cette ébauche. Cette détermination se fait à partir d'une aube modèle réalisée avec un élastomère permettant le développement à chaud de l'aube côté extrados. Ce développement, effectué sur aube modèle, donne le format de tôle capable de l'ébauche ainsi que les épaisseurs selon un quadrillage prédéterminé. Le procédé utilisé est le suivant:

- emboutissage à chaud de la tôle capable ;

- rechargement automatique inox par feuillard des zones de l'aube soumises à la cavitation ;

- formage final.

Notons que les déformations obtenues au cours du rechargement ont peu d'importance puisque le formage final est effectué en dernier lieu.

L'épaisseur de la soudure des aubes sur le plafond peut être de l'ordre de $180 \mathrm{~mm}$.

\subsection{Structures porteuses}

Les groupes hydro-électriques sont des machines pouvant présenter des masses tournantes considérables (plusieurs milliers de tonnes) et mobiliser des efforts très importants, tant au niveau hydraulique qu'électrique. Leurs structures porteuses doivent donc être étudiées avec le plus grand soin.

\subsubsection{Ligne d'arbres - Paliers}

Les turbomachines de grande puissance sont généralement à axe vertical et de ce fait, leurs paliers jouent un rôle essentiellement de guide. En règle générale, ils sont assez peu chargés en marche normale mais peuvent supporter des efforts pouvant atteindre, pour certains types de machines, plusieurs centaines de milliers de daN lors des déclenchements ou à l'emballement.

Les paliers sont à lubrification hydrodynamique. Comme nous allons examiner dans la suite celle des pivots, nous ne nous attarderons pas sur celle des paliers qui relève d'une analyse analogue. Les autres problèmes fondamentaux à résoudre relèvent d'une part, de l'approche précise des déformations thermo-élastiques relatives des organes porteurs et de l'arbre (palier s'effectuant sur de très grands diamètres, déformations locales des arbres creux; découplage des déformations des structures supports, etc.) et d'autre part, de la nécessité d'assurer une très grande rigidité d'appui, de façon à obtenir des vitesses critiques supérieures aux vitesses de fonctionnement. En effet, compte tenu de leur souplesse d'utilisateur et de leur grande plage de vitesse de rotation possible, les groupes hydro-électriques fonctionnent toujours en régime subcritique. Cette condition exige la réalisation de supports très rigides, alors que ces struc- 
tures peuvent atteindre, au niveau alternateur, des dimensions importantes (diamètre de fosse pouvant dépasser $20 \mathrm{~m}$ ).

Le problème de la stabilité des lignes d'arbres constitue l'une des questions majeures à résoudre dans le développement des groupes hydro-électriques de grandes puissances, à côté des problèmes liés aux vitesses d'écoulement pour les turbomachines de hautes chutes et au refroidissement des alternateurs résolvable quant à lui par un changement de technique (refroidissement à l'eau).

\subsubsection{Pivot}

L'accroissement des puissances des turbomachines hydrauliques et l'augmentation des poussées axiales qui en résulte, conduisent à des pivots dont la capacité de charge peut atteindre $5 \times 10^{6} \mathrm{daN}$. Cet organe constitue un des points sensibles du groupe, puisqu'il s'agit de supporter des charges de cette importance par un film d'huile de quelques centièmes de $\mathrm{mm}$ d'épaisseur, s'interposant entre des patins et une glace de plusieurs mètres de diamètre. L'équirépartition des charges entre les patins est assurée en montant ceux-ci sur des membranes souples remplies d'huile et reliées entre elles par un circuit fermé. L'échauffement par dissipation visqueuse du film de lubrification tend à faire chuter la capacité de charge par suite de la diminution de la viscosité et à produire un bombement du patin dû aux gradients thermiques. Ce problème doit donc être étudié avec le plus grand soin.

Un programme de calcul tridimensionnel, tenant compte des déformations du patin et des fuites latérales, a été développé. Il résoud :

- l'équation de la pression $p(r, \theta)$ dans le film, déduite des équations de Reynolds et de la continuité;

- l'équation de l'énergie dans le film tridimensionnel $r, \theta, z_{h}$;

- l'équation de la chaleur dans le patin en $r, \theta, z_{p}$; - l'équation de la chaleur dans le grain mobile en $r, z_{g}$

- I'équation d'équilibre mécanique du patin afin d'obtenir les déformations de celui-ci en $r, \theta$;

- l'équation d'équilibre du grain mobile pour obtenir des déformations radiales.

Les déformations thermo-élastiques du patin sont obtenues à l'aide d'un calcul par éléments finis.

Par ailleurs, au cours des itérations du calcul, la position du patin, compte tenu de l'emplacement du point d'appui, s'ajuste à une répartition des pressions correspondante. Il est tenu compte globalement de l'influence de l'espace et du volume d'huile situé entre les patins, sur la température du grain mobile. Grâce à cet outil, le comportement en service des parties actives du pivot est convenablement appréhendé. Les figures 6 et 7 montrent la répartition des pressions et la carte des déformations de la surface active d'un patin de pivoterie de forte charge.

En outre, pour les pivots de très forte charge, des patins d'un type nouveau, permettant de diminuer les deformations thermiques et d'augmenter les pressions spécifiques, ont pu être mis au point et testés industriellement avec la collaboration d'EDF sur un groupe de Salignac.

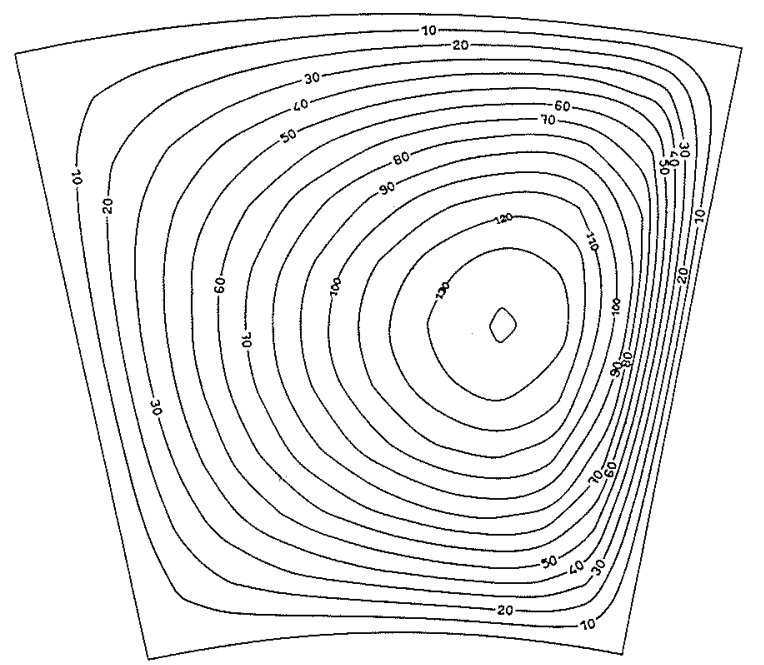

Figure 6. - Répartition des pressions calculée sur la face active d'un patin de pivoterie

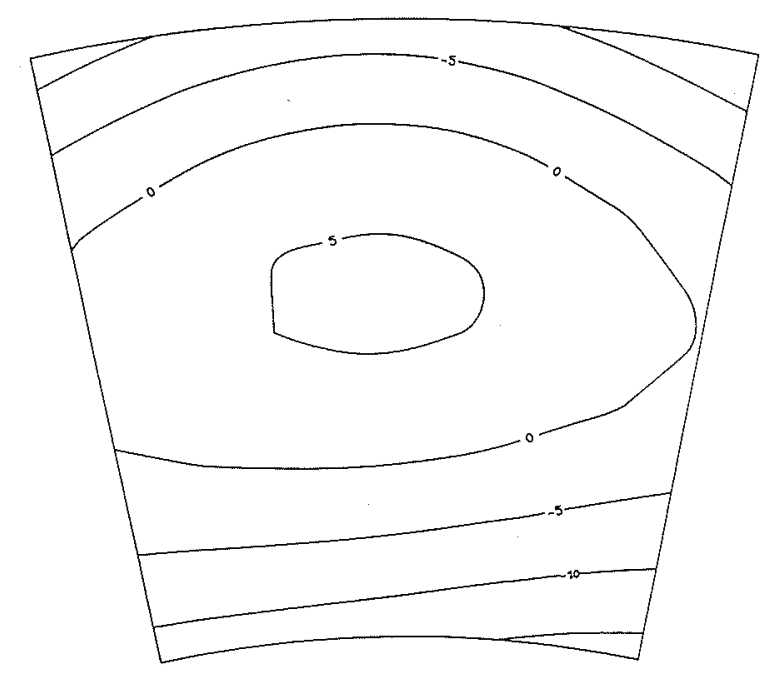

Figure 7. - Déformation en service de la face active d'un patin de pivoterie

Ces problèmes théoriques étant bien maîtrisés, nous soulignerons pour terminer, le soin qui doit être apporté à la réalisation des parties actives :

- tolérance de planéité de l'ordre de quelques centièmes de $\mathrm{mm}$ pour des grains mobiles de diamètre de l'ordre de $5 \mathrm{~m}$;

- voile dû au défaut de perpendicularité de la face active par rapport à l'axe de la ligne d'arbres à limiter également à quelques centièmes de $\mathrm{mm}$,

ceci afin d'éviter les mouvements dynamiques importants des patins.

\section{Conception assistée par ordinateur}

Grâce aux efforts de recherche engagés ces dernières années, la conception et la réalisation de turbomachines de grande puissance s'effectuent avec toute la confiance souhaitable. Il reste toutefois que l'ensemble des études exigées par ce type de machine, mobilise des moyens humains importants et nécessite un temps d'analyse 
prolongé. Aux enjeux techniques se superpose une concurrence internationale très vive, conduisant à la recherche constante d'une réduction des délais de réalisation parallèlement à l'élaboration d'un matériel de haute compétitivité. L'intervention massive de l'ordinateur dans tous les processus est indispensable pour relever ces défis.

NEYRPIC a donc développé depuis plusieurs années des logiciels spécifiques à ses besoins, sur la base d'un système (SYSTRID) pouvant manipuler des formes géométriques complexes.

Certains ont vocation d'aide au calcul scientifique, comme par exemple, le programme de maillage interactif MAO pour calcul par éléments finiș. D'autres sont relatifs à l'optimisation technico-économique des grands composants de turbines hydrauliques. Il s'agit là de programmes de CAO généralement interactifs, utilisant à la fois le système permettant de manipuler la géométrie, les modules de mécanique des fluides, ceux de mécanique des structures et des éléments de chiffrage économique. Ces logiciels concernent les avant-distributeurs et bâches, distributeurs, roues, aspirateurs et toutes les structures à caractère axisymétrique. Le mode de travail en interactif, avec dimensionnement simultané, a été retenu car il offre une souplesse très intéressante au niveau de la conception mécanique des structures. Il

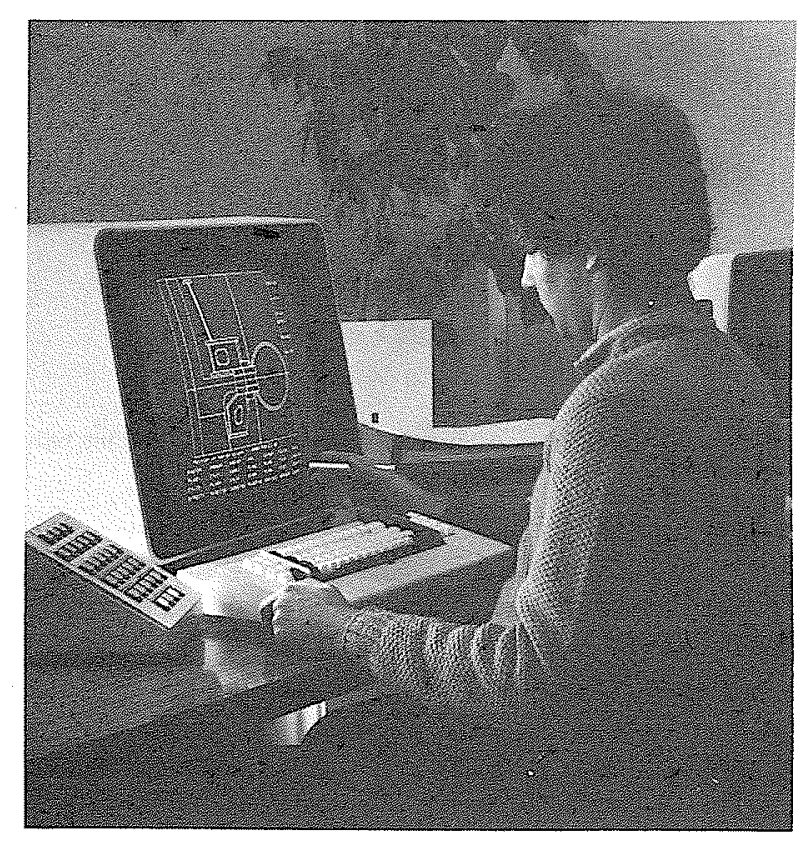

Figure 8. - Conception interactive d'un distributeur à l'aide du logiciel DISTURB développé par NEYRPIC s'adapte très bien au très grand particularisme des pièces en fonction des installations et a été rendu possible grâce aux nombreux programmes de calcul spécifiques développés par NEYRPIC depuis près de vingt ans.

$\mathrm{La}$ figure 8 illustre la conception interactive d'un distributeur de turbine-pompe.

Comme dans la plupart des logiciels de CAO que nous avons développés, l'analyse dimensionnelle peut être effectuée au fur et à mesure de la conception, grâce aux programmes de traitement des structures élémentaires de résistance de matériaux (coques, anneaux...) dont nous disposons, les éléments finis n'intervenant qu'au stade de la vérification.

Nous disposons ainsi de différents niveaux d'analyse, adaptés à la progression des phases de conception et permettant d'allier rapidité et fiabilité.

\section{Conclusion}

Le développement des turbomachines de grandes puissances a pu se poursuivre sans appréhension particulière grâce aux efforts de recherche fondés sur une meilleure approche des phénomènes physiques dans les écoulements et les matériaux.

Les ordinateurs, actuellement utilisables dans l'industrie sans requérir des investissements déraisonnables, offrent des possibilités de calculs qui permettent la mise au point de programmes performants, tant en ce qui concerne les écoulements que le comportement des structures.

Les techniques de soudage mises au point permettent de réaliser sur le site même des structures mécanosoudées de grandes dimensions et de fortes épaisseurs, reculant ainsi les limites liées aux processus de réalisation. De nouvelles conceptions élargissent le domaine des puissances réalisables. Ainsi se poursuit à pas mesurés mais sûrs, le développement des groupes hydro-électriques vers des puissances toujours plus élevées.

\section{Références bibliographiques}

[1] EREMEEF L.R. et PHILIBERT R. - Modélisation quasi tridimimensionnelle des écoulements dans les turbomachines hydrauliques, Journées S.H.F., novembre 1982.

[2] CASACCI S., LOURDEAUX B., WEGNER M. - Comportement dynamique des avant-distributeurs de grandes turbines Francis, Symposium A.I.R.H. - Amsterdam - Septembre 1982.

[3] CASACCI S., CAILLOT G., GIBERT R.J. - Vibrations des structures en forme de plaques et de coques en présence d'un milieu fluide incompressible, La Houille Blanche $n^{\circ} 1 / 2$ 1980. 